\title{
On the Joint Synchronization of Clock Offset and Skew in PBS-Protocol
}

\author{
Wenjing Liu ${ }^{1,}$, Zhi Wang ${ }^{2, b}$ \\ ${ }^{1}$ State Key Laboratory of Industrial Control Technology, Zhejiang University, Hangzhou, 310027 , \\ China \\ ${ }^{2}$ State Key Laboratory of Industrial Control Technology, Zhejiang University, Hangzhou, 310027, \\ China \\ aemail: liuwj9990122@126.com, bemail: wangzhizju@gmail.com
}

Keywords: Time Synchronization; Wireless Senor Networks; Clock Skew/Offset Estimation

\begin{abstract}
Joint estimation of clock offset and skew is a very important research for time synchronization. In this paper, we develop a set of energy-efficient clock synchronization algorithms of Wireless Senor Networks(WSNs) based on Pairwise Broadcast Synchronization Protocol(PBS). We formulate the clock offset and skew estimation problem as a linear least squares problem using auxiliary variables and solve the problem by LS and WLS algorithm and obtain the closed-form solution. Then, we compare the mean square error (MSE) of proposed LS, WLS algorithm and MLE. Finally, simulation results demonstrate that the proposed LS algorithm, WLS algorithm are low complexity with the estimation of WLS algorithm achieves higher accuracy.
\end{abstract}

\section{Introduction}

Clock synchronization among different nodes, which have their own autonomous clocks, is a key component of a wireless sensor network(WSN). Many coordinated applications require a common time frame for the entire network such as node localization[1], data fusion, sleeping and wake-up coordination, time-based channel sharing and scheduling, and other time-based tasks. However, every individual node in a WSN has its own clock. Different clocks drift from each other due to imperfections in the oscillator, aging, digital circuit design and other environmental variations. Also, sensor nodes are usually powered with just a battery. Thus, all tasks of a WSN, including synchronization, should be carefully performed to ensure longer operating lifetime. For synchronization, this means to minimize the number of transmissions between nodes during which the time-stamps are recorded.

The recent synchronization mechanisms can be roughly divided into two categories: 1) point to point synchronization protocols, such as two-way message exchange mechanism(TPSN) and one-way message mechanism, 2) broadcast synchronization protocols, such as pairwise broadcast synchronization (PBS) and reference broadcast synchronization (RBS).

Nohet al.[2] study the estimation of clock skew and offset in two-way message exchange mechanism. Then Leng[3] and Wu propose three estimators for clock skew and offset by assuming a Gaussian delay model and an unknown fixed delay. However, because of the broadcast property of the communication of a WSN, a two-way message exchange synchronization mechanism essentially leads to large communication overhead. And the inactive nodes do not make full use of the message received. Specifically, when two sensors are adopting a two-way message exchange synchronization mechanism, the sensors located in the common transmission range of these two nodes can also overhear the messages (Fig.1) and should be able to synchronize its own clock to some extent.

As for broadcast synchronization protocols, Noh etal. first proposed PBS[4]under a Guassian delay model. Afterwards, Chaudhariet al.[5] apply PBS to an exponential delay model and derive the MVUE and MLE of clock offset. Djamel[6]proposed a Relative Referenceless Receiver Receiver protocols and eliminated the single point of failure (reference)shortcomings of RBS. Xuanyu Cao[7] estimated joint clock skew and offset under exponential delay mode.

But PBS involves one more node in the analysis procedure when compared to two-way message 
exchange mechanism and the introduction of clock skew usually makes the problem much more complicated. So the estimation was complexity and nonlinear, thus is not more energy-efficient. In this paper, we formulate the clock offset and skew estimation problem as a linear least squares problem using auxiliary variables.

To summarize, the main contributions of this paper are as follows.

1) We consider a joint estimation of clock skew and offset by invoking PBS, and model the message exchange. It is energy -efficient.

2) We propose a low complexity LS algorithm and a WLS algorithm, also give the closed-form solution.

3) Compared with MLE, the WLS algorithm achieves higher accuracy.

The following of this paper is organized as follows. The clock mode is described In Section II. The time stamp exchange model based on PBS is given in Section III .And the low complexity LS algorithm and WLS algorithm are proposed in Section IV. The simulation results are shown in section $\mathrm{V}$. The conclusions are drawn at the end of this paper.

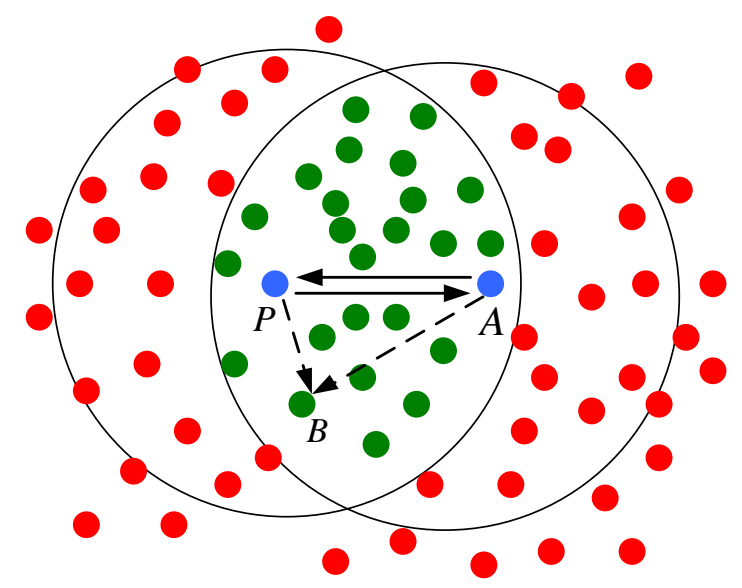

Fig.1A WSN with two active nodes $P$ and $A$ exchanging message with inactive node like $B$ around

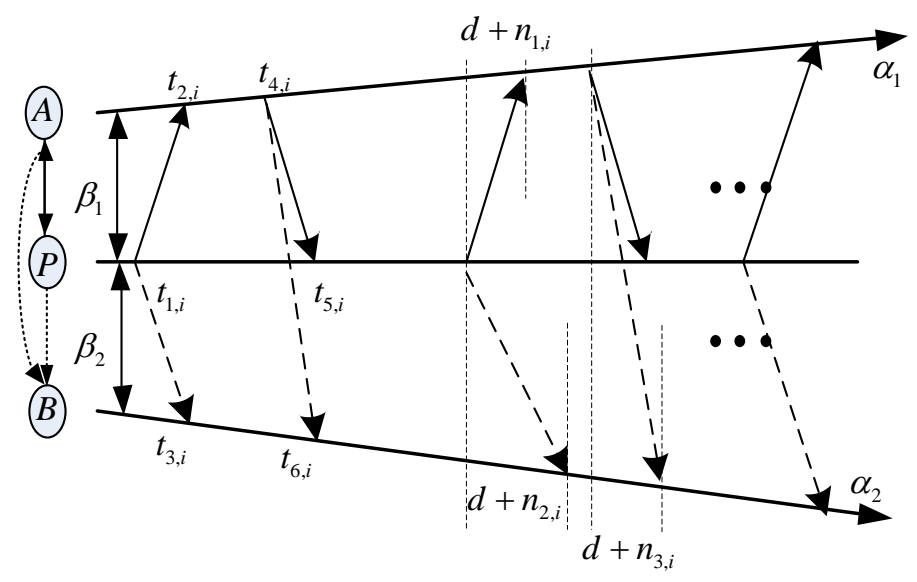

Fig2 Clock synchronization model of PBS

\section{A clock model}

Assume Node $A$ needs to synchronize with Node $P$ as shown in Fig.1. The clock values of the two nodes are $t_{p}=g_{p}(t) \quad t_{a}=g_{a}(t)$

respectively, where $t$ is the ideal global time. Here, $g_{p}$ and $g_{a}$ are continuous, differentiable, and strictly increasing functions, since we assume clocks can neither stop nor run backwards. No other constraints are required on the functions $g_{p}$ and $g_{a}$. For example, they can be linear functions, 
quadratic functions or even polynomials with higher orders[8]. And in this paper, we adopt a liner clock model

$$
t_{p}=\alpha_{p} t+\beta_{p} \quad t_{a}=\alpha_{a} t+\beta_{a}
$$

where $\alpha \in R_{+}$and $\beta \in R_{+}$are the clock skew and clock offset, $t$ is the ideal global time.

When Node $A$ is synchronized with Node $P$. Node $P$ is the reference node. We have

$$
t_{a}=\frac{\alpha_{a}}{\alpha_{p}} t_{p}+\alpha_{a}(d+n)+\beta_{a}-\frac{\alpha_{a}}{\alpha_{p}} \beta_{p}
$$

Where $d$ is the deterministic delay, and $n$ is the nondeterministic link delay. For the discussion in this paper, we also assume that the deterministic part of link delays $d$ is unknown but the same. This is because the nodes in a WSN usually share the same hardware specifications and characteristics and hence undergo similar transmission, reception, encoding, decoding, and byte alignment times.

As for nondeterministic link delay, there are different probability density function models for the random delays based on different justifications and applications, including Gaussian, exponential, Gamma, Weibull and log-normal[9].In[10], the chi-squared test showed that the variable portion of delays can be modeled as Gaussian distributed random variables with $99.8 \%$ confidence. In this paper, we can assume that the nondeterministic link delay $n$ is independent identically distributed Gaussian random variables.

When we have assumed $\alpha_{p}=1, \beta_{p}=0$, equation (3) becomes

$$
t_{a}=\alpha_{a}\left(t_{p}+d+n\right)+\beta_{a}
$$

\section{III .Time stamp exchange model in PBS}

Consider a WSN consisting of some sensor nodes as shown in Fig.1, which dynamically select a master node through any master election algorithm. Node $P$ is selected as the reference node whose time is chosen as the reference time subsequently for the rest of that synchronization cycle. Node $P$ and node $A$ are synchronizing via a two-way message exchange mechanism. Then, all the nodes(e.g. Node $B$ ) in the checked region can receive a series of synchronization messages containing the information about the time stamps of the pairwise synchronization.

The procedure for the message round exchange is shown in Fig.2 and depicted as follows. Node $P$ transmits a synchronization signal with time stamps $t_{1, i}$. Then node $A$ receives this signal and gives it a time stamp $t_{2, i}$ according to its own clock. Afterwards, node $A$ transmits a synchronization signal back to node $P$ at times $t_{4, i}$ of its own clock. Then node $P$ receives the signal and records the time as a time stamp of $t_{5, i}$. Thus a message round is finished. As shown in Fig.1, there may exist several silent sensor nodes located in the common transmission range of node $P$ and $A$. Suppose that node $B$ is one of such nodes. Hence, when node $P$ and $A$ are exchanging messages, node $B$ also overhears them and performs some synchronization accordingly. Since node $B$ is inactive, i.e., node $B$ does not need to pay any energy for communication, this synchronization mechanism (PBS) turns out to be much more energy efficient than other point-to-point mechanisms. Specifically, as Fig.1 illustrates, node $B$ will receive the message sent by $P$ and $A$ and give them time stamps $t_{3, i}$ and $t_{6, i}$ respectively. Note that $B$ will also know the value of $t_{2, i}$ since node $A$ is required to send this information back to node $P$ in a two-way message exchange mechanism. In sum, node $B$ will get the value of $\left\{t_{1, i}, t_{2, i}, t_{3, i}, t_{4, i}, t_{6, i}\right\}_{i=1}^{N}$.

For node $B$, the aforementioned message exchange procedure can be represented as follows

$t_{2, i}=\alpha_{1}\left(t_{1, i}+d+n_{1, i}\right)+\beta_{1}$

$t_{3, i}=\alpha_{2}\left(t_{1, i}+d+n_{2, i}\right)+\beta_{2}$

$t_{6, i}=\frac{\alpha_{2}}{\alpha_{1}}\left(t_{4, i}+d+n_{3, i}\right)+\beta_{2}-\alpha_{2} \frac{\beta_{1}}{\alpha_{1}}$

Where $\alpha_{1}$ and $\beta_{1}$ represent the clock offset and clock skew of node $A$ with respect to node $P$, 
respectively; $\alpha_{2}$ and $\beta_{2}$ represent the clock offset and clock skew of node $B$ with respect to node $P$, respectively. The model has two more parameters when compared to a two-way message exchange mechanism and the introduction of clock skew makes the problem much more complicated.

The goal is to estimate $\left\{\alpha_{1}, \alpha_{2}, \beta_{1}, \beta_{2}, d\right\}$ based on the observation of a set of time stamps $\left\{t_{1, i}, t_{2, i}, t_{3, i}, t_{4, i}, t_{6, i}\right\}_{i=1}^{N}$ accurately with low computational complexity.

\section{Proposed clock synchronization algorithm}

In this section, the ML joint skew and offset estimator has been used widely. However, a five-dimensional search is needed to obtain the solution for the five parameter estimation, which is computationally expensive and not feasible in energy limited WSNs. Unfortunately, with the nonlinear term, no closed form can be availably expressed for $\left\{\alpha_{1}, \alpha_{2}, \beta_{1}, \beta_{2}, d\right\}$ using MLE.

Also, the likelihood function may be not convex or log-convex, so we cannot find effective numerical methods to guarantee the globally optimal $\left\{\alpha_{1}, \alpha_{2}, \beta_{1}, \beta_{2}, d\right\}$. One can adopt search algorithms such as Gaussian-Newton algorithm, Nelder-Mead simplex algorithm or Newton-Raphson to seek the optimal solutions by setting a reasonable initialization. In order to avoid the computationally expensive numerical search, in the third part, we propose a low-complexity LS estimator.

Dividing both sides of

$\frac{1}{\alpha_{1}} \square \lambda_{1}, \frac{\beta_{1}}{\alpha_{1}} \square \lambda_{2}, \frac{1}{\alpha_{2}} \square \lambda_{3}, \frac{\beta_{2}}{\alpha_{2}} \square \lambda_{4}$ we can get

$$
\begin{aligned}
& \lambda_{1} t_{2, i}=t_{1, i}+d+n_{1, i}+\lambda_{2} \\
& \lambda_{3} t_{3, i}=t_{1, i}+d+n_{2, i}+\lambda_{4} \\
& \lambda_{3} t_{6, i}=\lambda_{1} t_{4, i}-\lambda_{2}+d+n_{3, i}+\lambda_{4}
\end{aligned}
$$

By observing that the uplink and downlink undergo the same amount of deterministic delay, we can rewrite the original model by subtracting (9) from (10) and subtracting (8) from (10), and the modified model is given by

$$
\begin{aligned}
& \lambda_{1} t_{2, i}-\lambda_{3} t_{3, i}+\lambda_{4}-\lambda_{2}=n_{1, i}-n_{2, i} \\
& \lambda_{3}\left(t_{6, i}-t_{3, i}\right)-\lambda_{1} t_{4, N}+\lambda_{2}=-t_{1, i}+n_{3, i}-n_{2, i}
\end{aligned}
$$

Stacking all the time stamps in matrix form, we have

$$
\underbrace{\left[\begin{array}{cccc}
t_{2,1} & -1 & -t_{3.1} & 1 \\
-t_{4.1} & 1 & t_{6.1}-t_{3.1} & 0 \\
\vdots & \vdots & \vdots & \vdots \\
t_{2 . N} & -1 & -t_{3 . N} & 1 \\
-t_{4 . N} & 1 & t_{6 . N}-t_{3 . N} & 0
\end{array}\right]}_{H} \underbrace{\left[\begin{array}{c}
\lambda_{1} \\
\lambda_{2} \\
\lambda_{3} \\
\lambda_{4}
\end{array}\right]}_{X}=\underbrace{\left[\begin{array}{c}
0 \\
-t_{1} \\
\vdots \\
0 \\
-t_{1, N}
\end{array}\right]}_{b}+\underbrace{\left[\begin{array}{c}
n_{1,1}-n_{2,1} \\
n_{3,1}-n_{2,1} \\
\vdots \\
n_{1, N}-n_{2, N} \\
n_{3, N}-n_{2, N}
\end{array}\right]}_{n}
$$

Since $n_{1, i}, n_{2, i}$ and $n_{3, i}$ are i.i.d. Gaussian distributed with variance $\sigma^{2}, \quad\left(n_{1, i}-n_{2, i}\right) \square N\left(0,2 \sigma^{2}\right)$ and $\left(n_{3, i}-n_{2, i}\right) \square N\left(0,2 \sigma^{2}\right)$.And $H$ represents the observation matrix of size $2 \mathrm{~N}^{*} 4$. The Least squares estimator for $X$ is given by

$$
\hat{X}=\left(H^{T} H\right)^{-1} H^{T} b
$$

Consequently, using the LS estimators (14), Node $B$ can be synchronized to Node $P$ without any additional message transmissions. Also, all the other nodes in the checked region in Fig. 1 can be simultaneously synchronized to the parent node $P$, thus saving a significant amount of energy.

Also, consider that $n_{1, i}, n_{2, i}$ and $n_{3, i}$ are i.i.d. Gaussian distributed with variance $\sigma^{2}$, we can solve 
the problem by WLS

$$
\hat{X}_{1}=\left(H^{T} C_{e}^{-1} H\right)^{-1} H^{T} C_{e}^{-1} b
$$

Where $C_{e}$ is the Covariance matrix of $n_{1, i}, n_{2, i}$ and $n_{3, i}$. Parameters can be calculated by solving

$$
\hat{\alpha}_{1}=\frac{1}{\hat{\lambda}_{1}}, \hat{\beta}_{1}=\hat{\lambda}_{1} \hat{\lambda}_{2}, \hat{\alpha}_{2}=\frac{1}{\hat{\lambda}_{3}}, \hat{\beta}_{2}=\hat{\lambda}_{3} \hat{\lambda}_{4}
$$

\section{Simulations}

In this section, simulation results are also presented to compare the effectiveness of the proposed LS clock synchronization algorithm, WLS clock synchronization algorithm and MLE.

In the simulations, the time skew is randomly drawn from [0.98, 1.02], and the time offset is randomly drawn from $[-10,10] \mathrm{ns}$. And the deterministic delay $d$ is randomly drawn from $(0,10] \mathrm{ns}$. The nondeterministic link delays are independent and identically distributed (i.i.d.) Gaussian random variables with zero mean and variance $\sigma^{2}$ drawn from $[0.5,5]$. Besides, the interval between the two-way timing messages, in terms of the reference time $t$, is assumed to be around 5s.The RMSEs of the time skew and time offset estimations are defined as $\sqrt{E\left\{\left(\alpha_{1}-\hat{\alpha}_{1}^{2}\right)^{2}\right\}}, \sqrt{E\left\{\left(\alpha_{2}-\hat{\alpha}_{2}^{2}\right)^{2}\right\}}$ and $\sqrt{E\left\{\left(\beta_{1}-\hat{\beta}_{1}^{2}\right)^{2}\right\}}, \sqrt{E\left\{\left(\beta_{2}-\hat{\beta}_{2}^{2}\right)^{2}\right\}}$, respectively. The number of observations(sets of time stamps) $N$ is randomly drawn from [5, 40]. All simulation results are average of 1000 independent runs.

Fig. 3 shows MSEs for estimation of the clock skew and offset as a function of $N$. For the clock skew estimation, MLE and WLS performs better than LS especially when $N$ is small. Also, WLS achieve a litter better than MLE. Simulations shows that three estimator perform similarly when $N$ is larger than 20.

Fig. 4 shows MSEs for estimation of the clock skew and offset as a function of $\sigma$ versus time stamps $N=20$. Simulations demonstrate that the nondeterministic link delay make magnitudes greater than the required precision of time synchronization. And clock skew and estimation get worse when the $\sigma$ is larger.
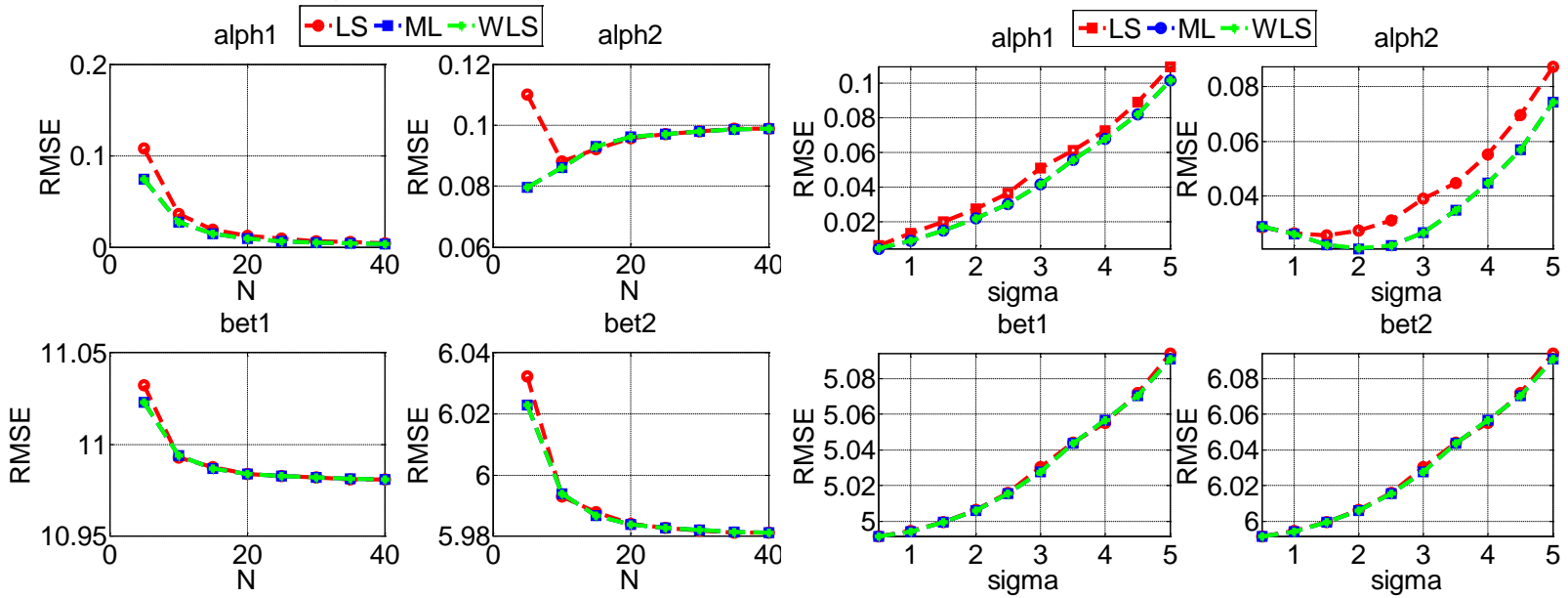

Fig.3. MSEs for estimation of the clock skew and offset versus variance $\sigma^{2}=1$

Fig.4. MSEs for estimation of the clock skew and offset versus time stamps $N=20$

\section{Conclusion}

In this paper we have proposed two energy-efficient algorithms for joint clock skew and offset based on PBS. The proposed algorithm involves auxiliary variables that leads to a closed form solution, and can very closely approach the LS and WLS solution in estimation performance at a 
significantly reduced computational complexity. We analyze and simulate the estimation performance of the proposed algorithms, and evidently demonstrate its superior, and achieve higher higher accuracy with low computational complexity than MLE.

\section{Acknowledgement}

This work was supported in part by the National Natural Science Foundation of China (No.NSFC61273079, No.NSFC11174316), in part by the Open Research Project of the State Key Laboratory of Industrial Control Technology, Zhejiang University(No.ICT1328, No.ICT1329) and in part by Key Laboratory of Wireless Sensor Network \& Communication of Chinese Academy of Sciences(No.WSNC2011001).

\section{References}

[1] Shao H J, Zhang X P, Wang Z. Efficient Closed-Form Algorithms for AOA Based Self-Localization of Sensor Nodes Using Auxiliary Variables[J]. Signal Processing, IEEE Transactions on, 2014, 62(10): 2580-2594.

[2] K. Noh, Q. Chaudhari, E. Serpedin, and B. Suter, Novel clock phase offset and skew estimation two-way timing message exchanges for wireless sensor networks, IEEE Trans. Commun., vol. 55, no. 4, pp. 766-777, Apr. 2007

[3] M. Leng and Y.-C. Wu, On clock synchronization algorithms for wireless sensor networks under unknown delay, IEEE Trans. Veh.Technol.,vol. 59, no. 1, pp. 182-190, Jan. 2010.

[4] K.-L. Noh, E. Serpedin, and K. A. Qaraqe, A new approach for time synchronization in wireless sensor networks: pairwise broadcast synchronization, IEEE Trans. Wireless Commun., vol. 7, no. 9, pp. 3318-3322, Sept. 2008

[5] Q. M. Chaudhari, E. Serpedin, and K. Qaraqe, Some improved and generalized estimation schemes for clock synchronization of listening nodes in wireless sensor networks, IEEE Trans. Commun.,vol. 58, no.1, pp. 63-67, Jan. 2010

[6] Djenouri D. : Relative Referenceless Receiver/Receiver Time Synchronization in Wireless Sensor Networks[J]. Signal Processing Letters, IEEE, 2012, 19(4): 175-178.

[7] Cao X, Yang F, Gan X, et al. Joint estimation of clock skew and offset in pairwise broadcast synchronization mechanism[J]. Communications, IEEE Transactions on, 2013, 61(6): 2508-2521.

[8] W. C. Lindsey, F. Ghazvinian, W. C. Hagmann, and K. Dessouky, Network synchronization, Proceedings of IEEE, vol. 73, no. 10, pp.1445-1467, Oct. 1985

[9] C. Bovy, H. Mertodimedjo, G. Hooghiemstra, H. Uijterwaal, and P. Mieghem, Analysis of end-to-end delay measurements in Internet, in Proc. Passive and Active Measurements Workshop, Mar. 2002, pp.26-33

[10] J. Elson, L. Girod, and D. Estrin, Fine-grained network time synchronization using reference broadcasts, inProc. Fifth Symposium on Operating System Design and Implementation, Dec. 2002. 